\title{
Endovascular Aortic Aneurysm Repair during the Early Days of the COVID-19 Outbreak
}

\author{
Kemal Karaarslan, Ayse Gul Kunt, Burcin Abud \\ Department of Cardiovascular Surgery, Health Sciences University, Izmir, Turkey
}

\section{ABSTRACT}

Introduction: We report the results of endovascular aortic repair (EVAR) in three patients during the COVID-19 pandemic. Materials and Methods: Three patients were diagnosed with abdominal aortic aneurysm. All three patients were male and aged 68 years. The diameter of the aneurysm was larger than $65 \mathrm{~mm}$ and was considered suitable for EVAR. Thorax tomography was performed to exclude SARS-CoV-2 infection before the procedure. Results: We performed EVAR under general anesthesia. "Priority Level" was based on the guideline of the American College of Surgeons. The procedure was conducted in the angiography laboratory by taking advanced precautions. Intensive care admission was avoided. The postoperative period was uneventful, and all patients were discharged without any condition associated with COVID-19. There were no mortality, rupture, secondary intervention, major adverse event, limb occlusion, and 60-day readmission. Conclusions: During the pandemic, EVAR can be performed for symptomatic abdominal aortic aneurysm with a diameter of more than $65 \mathrm{~mm}$. Thorax tomography is safe to exclude SARS-CoV-2 infection. However, tomographic angiography to monitor patients may be difficult during the pandemic.

Keywords: abdominal aortic aneurysm, endovascular aortic repair, thorax tomography, COVID-19

\section{ARTICLE HISTORY}

Received: November 24, 2020

Accepted: March 1, 2021

\section{CORRESPONDENCE}

\section{Ayse Gul Kunt}

Guney Mahallesi 1140/1, Sokak No:11

Yenisehir, Konak, Izmir, Turkey

Tel: +90 5324918912

Fax: +90 2324330756

E-mail: draysegulkunt@gmail.com

\section{INTRODUCTION}

Both the public and the health system have been seriously attacked globally by a new outbreak, COVID-19. The virus affected more than 4 million people and caused more than 278,000 deaths worldwide by early May 2020. ${ }^{1}$ In Turkey, it was reported that the total number of confirmed cases was 139,000 , which resulted in over 3,800 deaths. ${ }^{2}$ The Ministry of Health of the Republic of Turkey had assigned pandemic hospitals in all cities. Healthcare professionals were directed to a new working order under the name of "flexible work". Elective procedures and operations were postponed. The shift system was applied even dur- ing daytime hours. Emergency cases were carried out by shift physicians. When necessary, the surgeon on call was summoned for critical and complex cases that required a larger surgical team.

Abdominal aortic aneurysm (AAA) is a common public health problem. ${ }^{3}$ Death is caused most frequently by rupture, the risk increasing concomitantly with increasing aortic diameters. ${ }^{4}$ Death occurs in approximately $1 \%$ of male patients over 65 years of age..$^{5}$ It is well known that patients over 50 years of age with concomitant diseases are also at risk of severe or critical COVID-19 pneumonia. ${ }^{6}$ Thorax computed tomography (CT) is used for the diagnosis of COVID-19 pneumonia. ${ }^{7}$ 
The purpose of this article is to discuss the importance of thorax CT before endovascular aortic repair (EVAR) treatment during the COVID-19 pandemic.

\section{MATERIALS AND METHODS}

\section{PATIENTS}

This study was carried out in the highest volume primary care pandemic hospital in Izmir. The Clinical Research Ethics Committee approved the analysis and the publication of the data. The patients and the institution agreed with the study, and all study procedures were in line with the principles stipulated in the Declaration of Helsinki. A "Priority Level" based on the guideline of the American College of Surgeons was applied. Acceptable postoperative death risk for both open surgery and endovascular treatment was discussed with an anesthesiologist and a cardiologist. All patients provided written informed consent.

The study included three male patients with an average age of 68 years. The diagnosis was established using computed tomography angiography (CTA). The mean aneurysm diameter of patients was evaluated at $65 \mathrm{~mm}$ on CTA, and aortic morphology was assessed for compatible endograft location. The interference criterion was an aneurysm diameter of less than $55 \mathrm{~mm}$ relative to the CTA scan. The CTA data was used in order to plan and select the size and type of EVAR device (Figure 1).

\section{UNFITNESS FOR SURGERY AND PERIOPERATIVE RISK ASSESSMENT}

Although the evaluation of the American Society of Anesthesiologists (ASA) for perioperative risk is open to wide interpretations, it is thought to be important because its rating is simple. The assessment was made according to cardiac, pulmonary, and renal functions. ${ }^{8}$ Cardiac status was evaluated with conventional angiography and transthoracic echocardiography. Normally, myocardial infarction, onset of angina within three months, and unstable angina at rest and or night are not suitable for any surgical intervention. Patients with severe valve disease, significant arrhythmia, and uncontrolled congestive cardiac failure are referred to EVAR. Lung capacity was documented by respiratory test and X-ray. Open repair is not recommended for patients presenting with the symptoms, including shortness of breath at rest, FEV1 $<1.0 \mathrm{~L}, \mathrm{PO}_{2}<8.0$ $\mathrm{KPa}$, and $\mathrm{PCO}_{2}>6.5 \mathrm{KPa}$. Thorax tomography was planned to exclude COVID-19 pneumonia. Patients presenting with serum creatinine levels greater than $200 \mu \mathrm{mol} / \mathrm{L}$ are suitable for EVAR.

\section{OUTCOMES AND FOLLOW-UP}

The primary endpoint was all-cause death, including 60-day operative mortality. Patients also underwent CTA evaluation during the 1- and 3-month follow-up. The follow-up protocol of patients who undergo EVAR incudes

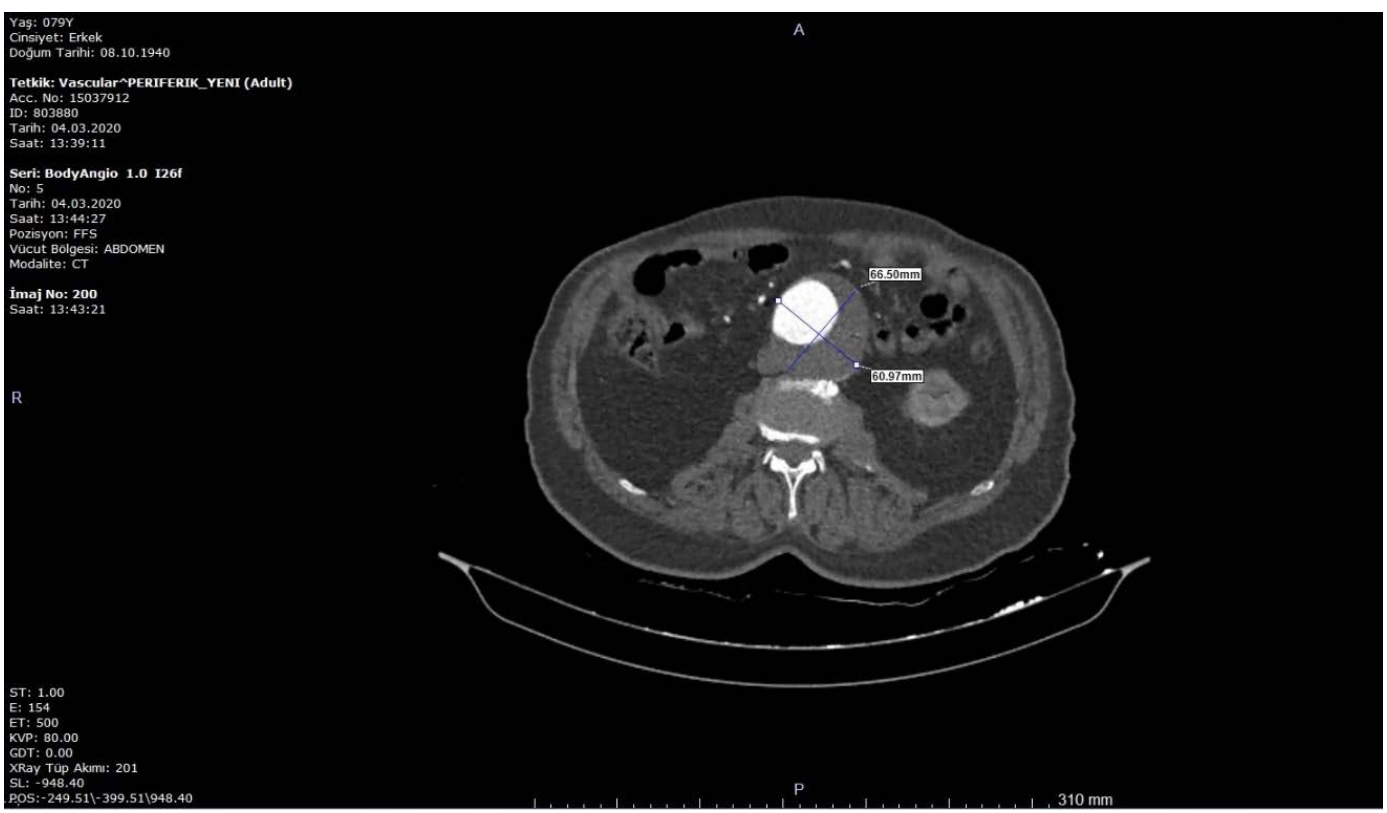

FIGURE 1. Tomographic angiography of abdominal aortic aneurysm before the procedure 
CTA examination annually in order to record any problems with the aneurysm or the implanted graft.

\section{RESULTS}

During the pandemic, three selected patients underwent EVAR of non-ruptured infrarenal AAA. The patients presented for abdominal pain and had been diagnosed with AAA. All were 68 years old males, with an AAA diameter of $65 \mathrm{~mm}$. The patients underwent EVAR using E-vita (Jotec, Hechingen, Germany). Comorbidities included coronary artery disease (1/3), hypertension (3/3), oral medicationdependent diabetes mellitus (1/3), and chronic obstructive pulmonary disease (2/3). All three patients were include in the ASA physical classification system class III, which includes individuals with a severe systemic disease that is not life-threatening such as arterial hypertension, diabetes, or chronic bronchopulmonary obstructive disease.

\section{PRE-PROCEDURAL COVID-19 SCREENING}

None of the patients complained of symptoms related to SARS-CoV-2 infection before the procedure. Preoperative laboratory studies were within normal limits. Thorax CT was performed for possible damage to the lungs, which may be caused by COVID-19.

Vascular access, stent-graft application, and stentgraft application were successful. Bilateral surgical access was provided. Conversion to open repair did not occur. The patients were monitored only for one night in the cardiovascular surgery unit. The follow-up was based on physical examination, face-to-face questioning, laboratory values, and phone calls. Questioning about infection was done in the postoperative period. Suspected contact was not reported in any patient. The patients were offered tomographic angiography, but they did not accept it. None of the patients had deceased during hospitalization or during follow-up, and there were no related complications such as rupture, secondary intervention, limb occlusion, or hospital readmissions.

\section{DISCUSSIONS}

COVID-19 pneumonia may progress to acute respiratory distress syndrome (ARDS) and multiple system failure accompanied by high mortality in critically ill patients (respiratory failure, septic shock, and multiorgan failure). ${ }^{9,10}$ Intensive care units have been reserved for COVID-infected patients, including cardiac surgery intensive care units. However, patients with vascular disease still require care.
Clinical guidelines for the treatment of vascular surgical patients have been published by the National Health Service (NHS) during the coronavirus pandemic. ${ }^{11}$ NHS has divided vascular surgery patients in several categories. The recommendation for an aortic aneurysm is as follows: prevent operative delay for symptomatic aortic aneurysm. The urgency of the aneurysm should be decided by a multidisciplinary team, depending on the size and symptoms of the aneurysm. Both the decision process and decision making should be well documented. The summary of all these recommendations can be explained as follows: minimum delay before surgery and minimum stay after surgery. Finally, more use of endovascular techniques is recommended to reduce critical care requirements as much as possible and speed up rehabilitation and in-hospital stay.

The American College of Surgeons has released a statement paper entitled "COVID 19: Elective Case Triage Guidelines for Surgical Care" on March 24, 2020. ${ }^{12}$ The college made a broad call to shorten "elective" surgical procedures as it is difficult to identify the medical emergency. Indeed, given the uncertainty regarding the effect of COVID-19 in the coming months, some postponed cases are at risk of re-emerging as more serious emergencies. Surgical procedures should be based not only on the risks associated with COVID-19 but also looking at the Elective Surgical Sharpness Scale (ESAS). ESAS has three headings: category (disease), condition (specifics of the disease), and tier class (what to do). The suggestion for symptomatic abdominal aortic aneurysm is "do not postpone" (tier class 3).

Currently, there is insufficient data and experience regarding cardiovascular surgery in COVID-19 infected patients. COVID-19 has very variable clinical manifestations without similar symptoms in patients. The clinical spectrum ranges from mild to critical presentations. ${ }^{13}$ The detection of SARS-CoV-2 patients has also proven difficult as the blood cell count and other laboratory parameters may be within normal limits. Although RT-PCR is considered the standard reference test, it has some disadvantages. Difficulties during sample collection and initial falsenegative results have led to using thorax $\mathrm{CT}$ as the first diagnostic tool. ${ }^{14}$ In the early stages, bilateral pneumonia and ground-glass opacity may be present on the chest CT before symptoms emerge. ${ }^{15}$ In $53.4 \%$ of asymptomatic patients, there are abnormalities on the chest $\mathrm{CT}$ at the time of admission. ${ }^{16}$ Chest CT was found to be completely normal in the patients presented in this case series.

Patients with pre-existing cardiovascular disease are prone to more serious complications of COVID-19, with increased mortality. ${ }^{17}$ Cardiovascular disease can also be an indicator of other mechanisms that affect the prognosis of 
old age and immune system dysregulation. Age is both a risk factor for cardiovascular disease and an essential determinant of COVID-19 mortality, which was found to be $14.8 \%$ in patients older than 80 years. Endovascular treatment does not have superiority over surgical treatment in terms of long-term survival, but it has some advantages in the short term. During the annual follow-up of 6,904 people, the EVAR group showed an early advantage in terms of AAA-related mortality that persisted for the first few years mainly due to lethal endograft ruptures: HR 0.92 ( $95 \%$ CI 0.57 to 1.49), $\mathrm{p}=0.73{ }^{18}$ The patients in this case series could have presented with vascular rupture if the procedure had been delayed for longer periods of time. Estimating the prognosis of such patients seems very difficult as there is insufficient data on COVID-19 associated with aortic aneurysms. With the help of the guidelines mentioned above, we decided that it would be better not to postpone the endovascular treatment of these patients. The three patients opted for general anesthesia. Early extubation reduces the risk of postoperative pneumonia, as well as the intensive care stay in patients with various associated conditions. ${ }^{19,20}$ Additional pulmonary support was not needed in our patients.

The length of hospital stay is variable in case of patients with open or endovascular aortic repair for aneurysms. In one study, the median procedure time (2.9 to 3.7 hours) and hospital stay (3 to 7 days) was shorter in patients in the endovascular repair group compared to open surgery. ${ }^{19}$ For our patients, the median procedure time was 3 to 3.6 hours, and hospital stay was only one night. This can be explained by the fact that the three subjects were prepared in outpatient conditions before the operation and hospitalized on the day of the procedure.

\section{CONCLUSIONS}

In conclusion, endovascular aortic repair may provide a short-term advantage in abdominal aortic aneurysms during the COVID-19 pandemic. Preoperative thorax CT is safer than RT-PCR to rule out COVID-19. In addition, patients can become positive during hospitalization, therefore, post-procedural tomographic angiography should be provided as soon as possible after the procedure, as both can evaluate the therapeutic success or complications, but also assess the pulmonary modifications that can become obvious during hospitalization.

\section{ACKNOWLEDGEMENT}

All personnel participating in the operation and in-patient care have used appropriate protective equipment (PPE) according to the guidelines recommended by the World Health Organization.

\section{CONFLICT OF INTEREST}

Nothing to declare.

\section{REFERENCES}

1. World Health Organization - Coronavirus disease (COVID-19) pandemic. Available at: https://www.who.int/emergencies/ diseases/novel-coronavirus-2019.

2. Turkey Republic Health Ministry - COVID-19 Bilgilendirme Platformu. Available at: https://covid19bilgi.saglik.gov.tr/tr/

3. Altobelli E, Rapacchietta L, Profeta VF, Fagnano R. Risk Factors for Abdominal Aortic Aneurysm in Population-Based Studies: A Systematic Review and Meta-Analysis. Int J Environ Res Public Health. 2018;15:2805. doi: 10.3390/ijerph15122805.

4. Institute for Health Metrics and Evaluation. Global Burden of Disease Study. Available at: http//www. healthmetricsandevaluation.org/gbd/visualizations/gbdcause-patterns

5. Thompson SG, Brown LC, Sweeting MJ, et al. Systematic review and meta-analysis of the growth and rupture rates of small abdominal aortic aneurysms: implications for surveillance intervals and their cost-effectiveness. Health Technol Assess. 2013;17:1-118. doi: 10.3310/hta17410.

6. The Novel Coronavirus Pneumonia Emergency Response Epidemiology Team. The epidemiological characteristics of an outbreak of 2019 novel coronavirus diseases (COVID-19): China, 2020. Chinese Center for Disease Control and Prevention. 2020;2:113-122.

7. World Health Organization. WHO Director-General's opening remarks at the media briefing on COVID-19 - March 112020. Available at: https://www.who.int/director-general/speeches/ detail/who-director-general-s-opening-remarks-at-themedia-briefing-on-covid-19---11-march-2020

8. Brown LC, Epstein D, Manca A, Beard JD, Powell JT, Greenhalgh RM. The UK Endovascular Aneurysm Repair (EVAR) trials: design, methodology and progress. Eur J Vasc Endovasc Surg. 2004;27:372-381. doi: 10.1016/j.ejvs.2003.12.019.

9. WHO. Novel coronavirus (2019-nCoV) situation Report-1, 2020. Available at: https:// www.who.int/docs/defaultsource/coronaviruse/situation-reports/20200121-sitrep-12019-ncov.pdf?sfvrsn=20a99c10_4

10. China CDC. The epidemiological characteristics of an outbreak of 2019 novel coronavirus diseases (COVID-19) - China, 2020. Chinese Center for Disease Control and Prevention. 2020;2:113-122.

11. National Health Service. Clinical guide for the management of vascular surgery patients during the Coronavirus pandemic. Available at: https://www.england.nhs.uk/coronavirus/ secondary-care/other-resources/specialty-guides/

12. American College of Surgeons. COVID-19 Guidelines for Triage of Vascular Surgery Patients. Available at: https://www.facs. org/covid-19/clinical-guidance/elective-case/vascularsurgery.

13. Liang WH, Guan WJ, Li CC, et al. Clinical characteristics and outcomes of hospitalised patients with COVID-19 treated 
in Hubei (epicenter) and outside Hubei (non-epicenter): A Nationwide Analysis of China. Eur Respir J. 2020;55:2000562. doi: 10.1183/13993003.00562-2020.

14. Gunduz Y, Ozturk MH, Tomak Y. The usual course of Thorax CT indings of Covid-19 infection and When should control Thorax CT scan? Turk J Med Sci. 2020;50:684-686. doi: 10.3906/sag-2004-293.

15. Guneyli S, Atceken Z, Dogan H, Altinmakas E, Atasoy KC. Radiological approach to COVID-19 pneumonia with an emphasis on chest CT.Diagn Interv Radiol. 2020;26:323-332. doi: 10.5152/dir.2020.20260.

16. $\mathrm{Xu} \mathrm{T}$, Huang $\mathrm{R}, \mathrm{Zhu} \mathrm{L}$, et al. Epidemiological and clinical features of asymptomatic patients with SARS-CoV-2 infection. J Med Virol. 2020;92:1884-1889. doi: 10.1002/jmv.25944.

17. Li B, Yang J, Zhao F, et al. Prevalence and impact of cardiovascular metabolic diseases on COVID-19 in China. Clin
Res Cardiol. 2020;109:531-538. doi: 10.1007/s00392-02001626-9.

18. Brown LC, Powell JT, Thompson SG, Epstein DM, Sculpher MJ, Greenhalgh RM. The UK EndoVascular Aneurysm Repair (EVAR) trials: randomised trials of EVAR versus standard therapy. Health Technol Assess. 2012;16:1-218. doi: 10.3310/ hta16090.

19. Le CD, Lehman E, Aziz F. Development of Postoperative Pneumonia After Endovascular Aortic Aneurysm Repair is Associated with an Increased Length of Intensive Care Unit Stay. Cureus. 2019;11:e4514. doi: 10.7759/cureus.4514.

20. Lederle FA, Freischlag JA, Kyriakides TC, et al. Outcomes following endovascular vs open repair of abdominal aortic aneurysm: a randomised trial. JAMA. 2009;302:1535-1542. doi: 10.1001/jama. 2009.1426. 